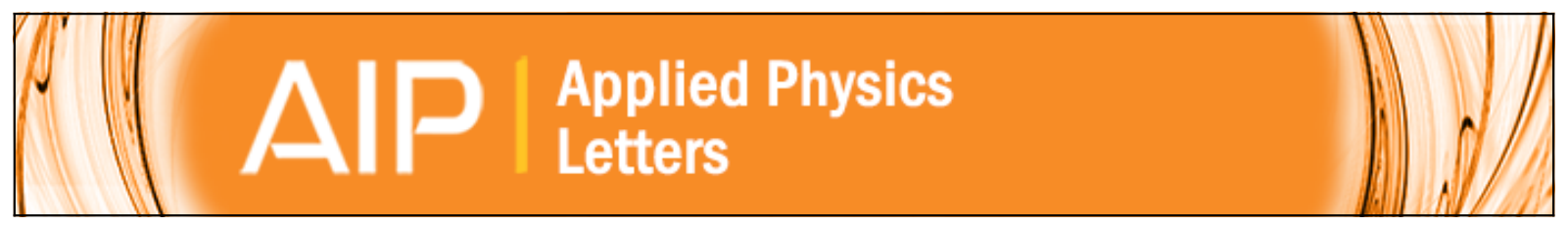

\title{
Smart photochromic gratings with switchable wettability realized by green-light interferometry
}

Elisa Mele, Dario Pisignano, Maria Varda, Maria Farsari, George Filippidis, Costas Fotakis, Athanassia Athanassiou, and Roberto Cingolani

Citation: Applied Physics Letters 88, 203124 (2006); doi: 10.1063/1.2198509

View online: http://dx.doi.org/10.1063/1.2198509

View Table of Contents: http://scitation.aip.org/content/aip/journal/apl/88/20?ver=pdfcov

Published by the AIP Publishing

\section{Articles you may be interested in}

Freezing of sessile water droplets on surfaces with various roughness and wettability

Appl. Phys. Lett. 104, 161609 (2014); 10.1063/1.4873345

X-ray grating interferometry with a liquid-metal-jet source Appl. Phys. Lett. 103, 091105 (2013); 10.1063/1.4819766

Sensitivity in X-ray grating interferometry on compact systems

AIP Conf. Proc. 1466, 293 (2012); 10.1063/1.4742307

Design aspects of X-ray grating interferometry

AIP Conf. Proc. 1466, 84 (2012); 10.1063/1.4742273

Photocontrolled wettability changes in polymer microchannels doped with photochromic molecules Appl. Phys. Lett. 91, 113113 (2007); 10.1063/1.2784191 


\title{
Smart photochromic gratings with switchable wettability realized by green-light interferometry
}

\author{
Elisa Mele, ${ }^{\text {a) }}$ Dario Pisignano, Maria Varda, ${ }^{\text {b) }}$ Maria Farsari, George Filippidis, \\ Costas Fotakis, ${ }^{\text {b) }}$ and Athanassia Athanassiou \\ Institute of Electronic Structure and Laser (IESL), Foundation for Research and Technology-Hellas \\ (FORTH), 71110 Heraklion, Crete, Greece \\ Roberto Cingolani \\ NNL, National Nanotechnology Laboratory of CNR-INFM, Università degli Studi di Lecce, \\ clo Distretto Tecnologico ISUFI, via Arnesano, I-73100 Lecce, Italy
}

(Received 14 January 2006; accepted 9 March 2006; published online 19 May 2006)

\begin{abstract}
We demonstrate the enhancement of the wetting properties of smart photochromic surfaces by a specifically developed, gentle interferometric patterning employing green light. We realized photochromic gratings with $2.5-10.0 \mu \mathrm{m}$ period by a blend consisting of a green-curable matrix and spiropyran molecules. The structured surfaces exhibit photocontrolled and reversible wettability, and enhanced hydrophilicity with respect to the native substrates. The dynamics of liquid spreading onto the gratings was also investigated, and the wetting behavior analyzed according to Wenzel's [Ind. Eng. Chem. 28, 988 (1936)] model for rough surfaces. These results indicate switchable gratings as promising functional components for microfluidics and modulation, and green-light interferometry as a reliable lithographic method for patterning organics without degradation or photochemical reactions. (C) 2006 American Institute of Physics.
\end{abstract}

[DOI: $10.1063 / 1.2198509$ ]

The wettability of solid surfaces by a liquid is a wellknown physical phenomenon, governed by both the chemical and the geometrical surface characteristics. ${ }^{1,2}$ Many studies have investigated the wetting behavior of a liquid onto artificial superhydrophobic surfaces, ${ }^{3-9}$ whose topology mimics the leaves of the lotus plant (Nelumbo nucifera ${ }^{10}$ ) and the wings of some insects (Pflatoda claripennis ${ }^{11}$ ). Moreover, different methods are reported for producing smart surfaces, able to change reversibly their wettability in response to a variety of external stimuli, such as light irradiation, ${ }^{12,13}$ electric fields, ${ }^{14,15}$ and thermal ${ }^{16}$ and chemical changes. ${ }^{17}$ These are particularly appealing for the realization of reversible switches with tunable wettability. ${ }^{18}$

Recently, photochromic spiropyrans were employed in the realization of photosensitive surfaces, that change reversibly their physical and chemical properties by the use of the light. ${ }^{12,19}$ Photochromism is a reversible transformation of a chemical species, induced by electromagnetic radiation, between two forms having different absorption spectra. ${ }^{20}$ The corresponding reversible effects on dipole moment, surface energy, refractive index, and volume have numerous promising applications in switching ${ }^{21}$ devices for three-dimensional (3D) optical memories, ${ }^{22}$ modulators, ${ }^{23}$ holographic recording, ${ }^{24}$ microfluidics, ${ }^{19}$ photoswitches of protein activity, ${ }^{25}$ and actuators. ${ }^{26}$ For these reasons, lithographic approaches able to structure photochromic molecules, enabling their integration within functional devices without deterioration of their properties, are strongly desired.

In this letter, we demonstrate the enhancement of the wetting properties of smart photochromic surfaces by a specifically developed, gentle interferometric patterning em-

\footnotetext{
${ }^{a)}$ Electronic mail: elisa.mele@unile.it

b) Also at: University of Crete, Physics Department, 71003 Heraklion, Crete, Greece.
}

ploying green light. We realized photochromic gratings with a period in the range of $2.5-10.0 \mu \mathrm{m}$ by structuring a suitable blend consisting of a green-curable matrix and spiropyrans, exhibiting photocontrolled and reversible wettability, and enhanced hydrophilicity (contact angle reduced by more than $20 \%$ ).

A highly coherent, cw Nd: $\mathrm{YVO}_{4}$ (yttrium vanadate) laser $(\lambda=532 \mathrm{~nm})$ was used for generating interferometric gratings [Fig. 1(a)]. As target organic films we employed optimized polymeric blends consisting of $1^{\prime}, 3^{\prime}$-dihydro$1^{\prime}, 3^{\prime}, 3^{\prime}$-trimethyl-6-nitrospiro [2H-1-benzopyran-2,2' $(2 \mathrm{H})$-indole] $\left(6-\mathrm{NO}_{2}\right.$-BIPS) in a photopolymerizable material with absorption maximum at $530 \mathrm{~nm} \cdot{ }^{27-29}$ The $6-\mathrm{NO}_{2}$ BIPS is initially in a closed, colorless, and nonpolar spiroform [Fig. 1(b)]. The absorption of ultraviolet (UV) radiation induces the photochemical cleavage of the $\mathrm{C}-\mathrm{O}$ bond in the

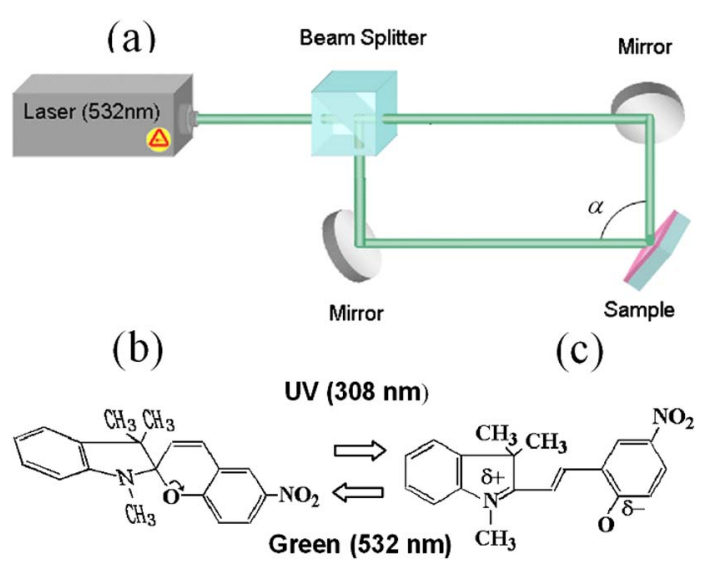

FIG. 1. (Color online) (a) Scheme of the interferometric setup. Reversible transformations between (b) the closed and (c) the open form of the photochromic molecule (6- $\mathrm{NO}_{2}$-BIPS and $\mathrm{MC}$, respectively), by irradiation with $\mathrm{UV}$ and green light. 


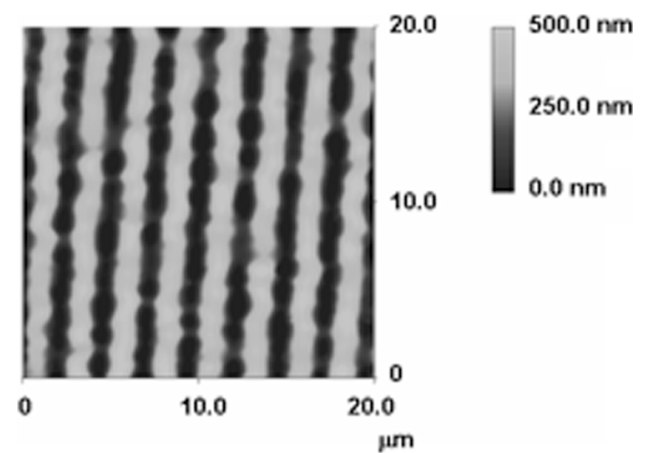

FIG. 2. (Color online) Planar view of the $2.5 \mu \mathrm{m}$ period grating imaged by atomic force microscopy.

spiropyran ring, i.e., the conversion into its isomeric open form, the highly polar trans-merocyanine [MC, Fig. 1(c) ]..$^{30}$ This absorbs at visible wavelengths. The photoconversion is reversible: the open (closed) form switches to the closed (open) one by absorbing green (UV) light. The used photopolymerizable system consisted of three components, namely, (i) a sensitizer dye, the eosin Y $\left(2^{\prime}, 4^{\prime}, 5^{\prime}, 7^{\prime}\right.$-tetrabromofluorescein disoltium salt), (ii) an amine molecule, methyldiethanolamine (MDEA), and (iii) pentaerythritol triacrylate (PETIA). The photopolymerization reaction is initiated through a radical-based process. ${ }^{29}$ In particular, eosin $\mathrm{Y}$ and MDEA are involved in the radical formation. Once the sensitizing dye absorbs the visible radiation, the amine can be oxidized by the triplet state of the dye, thus allowing the PETIA monomer to cross link.

The films were spin cast at $3000 \mathrm{rpm}$ for 2 min onto glass substrates. The resulting layer was completely cured by exposing it to $1.0 \mathrm{~W}$ laser beam for $20 \mathrm{~s}$, and another blend film was then deposited on the top. Then, the sample was exposed to the two interfering beams $(5 \mathrm{~s}$ at $0.4 \mathrm{~W})$, thus producing one-dimensional (1D) patterns, with 2.5, 5.0, and $10.0 \mu \mathrm{m}$ periods $(\Lambda)$ onto the second layer, according to the well-known expression $\Lambda=\lambda / 2 n \sin (\alpha / 2)$, where $n$ and $\alpha$ indicate the refractive index of the organic blend and the interference angle, respectively. The use of two superimposed layers allowed us to obtain textured surfaces with the same surface energy, both in the grooves and on the relief features. After exposure, the sample was washed in a bath of de-ionized water for $4 \mathrm{~h}$, in order to completely develop the grating, and dried by a nitrogen flux. The wettability switching of our gratings was checked by alternately exposing them by a $\mathrm{XeCl}$ laser operating at $308 \mathrm{~nm}$ (50 pulses of $30 \mathrm{~ns}$ at a fluence of $40 \pm 1 \mathrm{~mJ} / \mathrm{cm}^{2}$, Lambda Physik EMG201MSC), and by a Nd:YAG (yttrium aluminum garnet) laser, operating at $532 \mathrm{~nm}\left(100\right.$ pulses of $5 \mathrm{~ns}$ at $47 \pm 1 \mathrm{~mJ} / \mathrm{cm}^{2}$, B. M. Industries, series 5000).

Like mechanical approaches, such as nanoimprint ${ }^{31}$ and soft lithography, ${ }^{32}$ our green-light holography offers the cru-
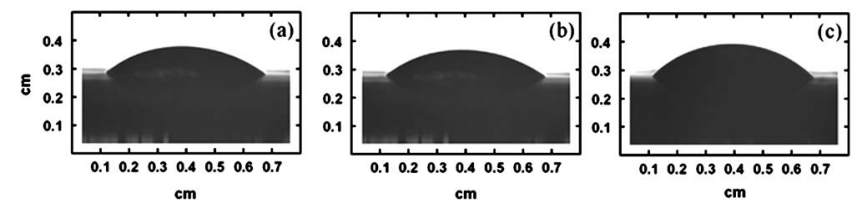

FIG. 3. Digital images of a water droplet placed on the patterned area collected after (a) $25 \mathrm{~min}$ for the $2.5 \mathrm{\mu m}$ neriod grating, (b) $80 \mathrm{~min}$ for the $5.0 \mu \mathrm{m}$ period one, and (c) $115 \mathrm{~min}$ for the $10.0 \mu \mathrm{m}$ period one.
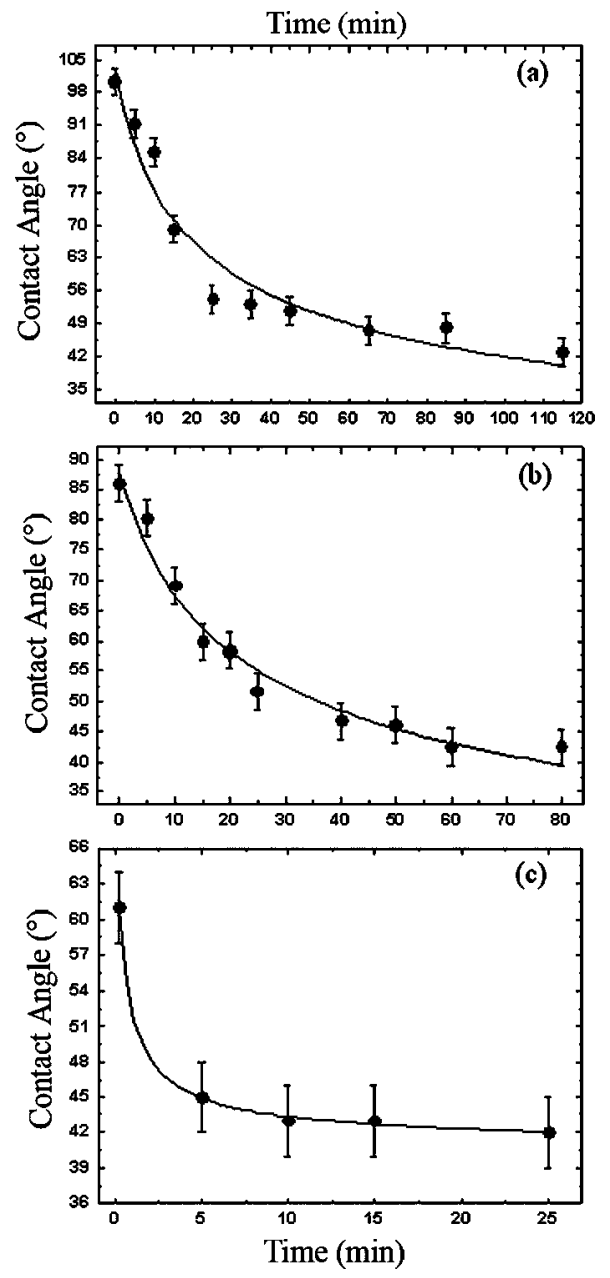

FIG. 4. Spreading dynamics of $3 \mu 1$ water droplet over the realized gratings: (a) $\Lambda=10.0 \mu \mathrm{m}$, (b) $\Lambda=5.0 \mu \mathrm{m}$, and (c) $\Lambda=2.5 \mu \mathrm{m}$. The black circles represent the experimental data, whereas the solid line is the fitting curve according to a power law.

cial advantage of structuring organic layers and realizing high-quality structures (Fig. 2) without degradation of the active medium during patterning. The optical properties of the photosensitive PETIA solution, and especially the absorption peak at $530 \mathrm{~nm}$, are ideal for blending with spiropyrans, which are transparent at this wavelength. Hence, during interferometry only the sensitizer dye absorbs, inducing the photopolymerization of the monomer, whereas 6- $\mathrm{NO}_{2}$-BIPS remains in the closed form without undergoing chemical modifications.

The realized patterns were first employed to investigate the influence of the topography on the wettability of the spiropyran (SP)/PETIA surfaces. For a droplet placed on a smooth flat substrate, the wetting properties are governed by the balance of the three interfacial tensions (solid vapor, $\gamma_{\mathrm{SV}}$, solid liquid, $\gamma_{\mathrm{SL}}$, and liquid vapor, $\gamma_{\mathrm{LV}}$ ) at the contact line, or equivalently, of the surface free energies for unit interfacial area, according to Young's law: $\gamma_{\mathrm{LV}} \cos \theta_{Y}=\gamma_{\mathrm{SV}}-\gamma_{\mathrm{SL}}$, where $\theta_{Y}$ is the contact angle. Topographical structuring alters the interfacial free energies, and the resulting contact angle $\theta_{W}$ is often related to $\theta_{Y}$ by Wenzel's equation: $\cos \theta_{W}=r \cos \theta_{Y}{ }^{2}$ where $r>1$ is the roughness factor, namely, the ratio of the actual area of a rough surface to the geometric projected area. According to Wenzel's model, the local geometry induces a decrement (increment) of the observed contact angle on a textured area, if the contact angle value for the smooth 


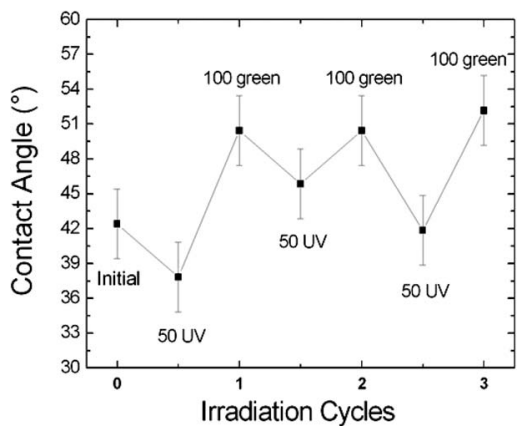

FIG. 5. Contact angle values ( $25 \mathrm{~min}$ after drop deposition) for three UVgreen irradiation cycles on a $2.5 \mu \mathrm{m}$ period grating.

surface is smaller (larger) than $90^{\circ}$. We measured for a flat sample a value of the contact angle of $(55 \pm 3)^{\circ}$, higher than those onto the textured regions: $(43 \pm 3)^{\circ}$ for the $10.0 \mu \mathrm{m}$ period, $(42 \pm 3)^{\circ}$ for the $5.0 \mu \mathrm{m}$ one, and $(42 \pm 3)^{\circ}$ for the $2.5 \mu \mathrm{m}$ one (Fig. 3). This is in agreement with Wenzel's theory.

In order to achieve a deeper insight into the wettability properties of our gratings, we investigated the temporal dynamics of the deposited drops. Evolution of the liquid spreading on very hydrophilic surfaces is usually described by Tanner's equation: ${ }^{33-35} \theta \propto(t+C)^{-n}$, where $C$ and $n$ are constants. The temporal dynamics of the drop spreading onto our gratings is well described by a Tanner law, with $n$ equal to $0.4 \pm 0.2$ for $\Lambda=10 \mu \mathrm{m}$ and $\Lambda=5 \mu \mathrm{m}$, and $0.7 \pm 0.1$ for $\Lambda=2.5 \mu \mathrm{m}$ (Fig. 4). The value of the exponent depends on the local topography, increasing upon increasing the surface roughness, i.e., upon decreasing the grating period. We conclude that the presence of the periodic corrugations strongly enhances the wettability of the SP/PETIA surfaces, inducing an increment of the hydrophilicity. Moreover, we observed that for the smallest period grating, $\theta$ reaches its stability condition more rapidly. This is an important indication for the design of fast commutating surface with switchable wettability.

We exploited the presence of the preserved photochromic molecules to induce controlled switching of the wetting properties in the realized gratings. The variation of the contact angle value during three subsequent UV-green cycles on the $2.5 \mu \mathrm{m}$ period grating is displayed in Fig. 5. The enhancement of the hydrophilic properties of the patterned surface upon UV irradiation, resulting in the decrease of the contact angle by $5^{\circ}-9^{\circ}$, is due to the conversion of 6- $\mathrm{NO}_{2}$-BIPS to polar MC. ${ }^{36}$ When the photoconversion to MC occurs, the solid-vapor interface tension increases and the sample surface becomes more hydrophilic. The green irradiation, instead, converts the molecules back to the initial form, and an increase of the contact angle up to $12^{\circ}$ is observed. However, the SP/PETIA system did not photoconvert back completely, the resulting hydrophobicity being therefore increased, with an increment of $\theta$ of about $8^{\circ}$ with respect to the initial value. We attribute this effect to the fact that the green pulses as well as the UV pulses induced a further polymerization of the PETIA matrix (highly sensitive at $532 \mathrm{~nm}$ and at the UV wavelengths), enhancing the molecular steric hindrance and thus reducing the efficiency of photoconversion.

In conclusion, we demonstrated the realization of smart photochromic gratings hosing the molecules, $6-\mathrm{NO}_{2}$-BIFS, by function-preserving green-light holography. The surfaces exhibit reversible, photocontrollable changes of the wetting properties, and enhancement hydrophilicity (with a contact angle decrease of more than 20\%) with respect to native substrates. These results indicate switchable gratings as promising functional components for microfluidic and modulation applications.

Two of the authors (E.M.) and (D.P.) acknowledge support from the Marie Curie Research Training Networks, HPMT-CT-2000-00201 and MRTN-CT-2003-505138, respectively (EU sixth Framework Program).

${ }^{1}$ D. Myers, Surfaces, Interfaces and Colloids (Wiley-VCH, Weinheim, 1999).

${ }^{2}$ R. N. Wenzel, Ind. Eng. Chem. 28, 988 (1936).

${ }^{3}$ S. Shibuichi, T. Onda, N. Satoh, and K. Tsujii, J. Phys. Chem. 100, 19512 (1996).

${ }^{4}$ N. A. Patankar, Langmuir 20, 8209 (2004).

${ }^{5}$ K. K. S. Lau, J. Bico, K. B. K. Teo, M. Chhowalla, G. A. J. Amaratunga, W. I. Milne, G. H. McKinley, and K. K. Gleason, Nano Lett. 3, 1701 (2003).

${ }^{6}$ E. Martines, K. Seunarine, H. Morgan, N. Gadegaard, C. D. W. Wilkinson, and M. O. Riehle, Nano Lett. 5, 2097 (2005).

${ }^{7}$ R. Furstner, W. Barthlott, C. Neinhuis, and P. Walzel, Langmuir 21, 956 (2005).

${ }^{8}$ M. Miwa, A. Nakajima, A. Fujishima, K. Hashimoto, and T. Watanabe, Langmuir 16, 5754 (2000).

${ }^{9}$ D. Oner and T. J. McCarthy, Langmuir 16, 7777 (2000).

${ }^{10}$ W. Barthlott and C. Neinhuis, Planta 202, 1 (1997).

${ }^{11}$ G. S. Watson and J. A. Watson, Appl. Surf. Sci. 235, 139 (2004).

${ }^{12}$ A. Athanassiou, M. I. Lygeraki, E. Mele, D. Pisignano, K. Lakiotaki, M. Varda, C. Fotakis, R. Cingolani, and S. H. Anastasiadis, Langmuir 22, 2329 (2006).

${ }^{13}$ K. Ichimura, S. K. Oh, and M. Nakagawa, Science 288, 1624 (2000).

${ }^{14}$ M. W. J. Prins, W. J. J. Welters, and J. W. Weekamp, Science 291, 277 (2001).

${ }^{15}$ J. Lahann, S. Mitragotri, T. N. Tran, H. Kaido, J. Sundaram, I. S. Choi, S. Hoffer, G. A. Somorjai, and R. Langer, Science 299, 371 (2003).

${ }^{16}$ G. B. Crevoisier, P. Fabre, J. M. Corpart, and L. Leibler, Science 285, 1246 (1999).

${ }^{17}$ S. Minko, M. Muller, M. Motornov, M. Nitschke, K. Grundke, and M. Stamm, J. Am. Chem. Soc. 125, 3896 (2003).

${ }^{18}$ T. L. Sun, G. J. Wang, L. Feng, B. Q. Liu, Y. M. Ma, L. Jiang, and D. B. Zhu, Angew. Chem., Int. Ed. 43, 357 (2004).

${ }^{19}$ R. Rosario, D. Gust, M. Hayes, F. Jahnke, J. Springer, and A. A. Garcia, Langmuir 18, 8062 (2002).

${ }^{20}$ H. Bouas-Laurent and H. Durr, Pure Appl. Chem. 73, 639 (2001).

${ }^{21}$ F. M. Raymo and S. Giordani, Proc. Natl. Acad. Sci. U.S.A. 99, 4941 (2002).

${ }^{22}$ A. S. Dvornikov, J. Makin, and P. M. Rentzepis, J. Phys. Chem. 98, 6146 (1994).

${ }^{23}$ J.-W. Kang, J.-J. Kim, and E. Kim, Appl. Phys. Lett. 80, 1710 (2002).

${ }^{24}$ A. Akella, S. L. Sochava, and L. Hesselink, Opt. Lett. 22, 919 (1997).

${ }^{25}$ I. Willner, Acc. Chem. Res. 30, 347 (1997).

${ }^{26}$ A. Athanassiou, M. Kalyva, K. Lakiotaki, S. Georgiou, and C. Fotakis, Adv. Mater. (Weinheim, Ger.) 17, 988 (2005).

${ }^{27}$ S. Sainov, C. Ecoffet, and D. J. Lougnot, J. Mater. Sci.: Mater. Electron. 14, 855 (2003).

${ }^{28}$ C. Triger, C. Ecoffet, and D. J. Lougnot, J. Phys. D 36, 2553 (2003).

${ }^{29}$ A. Espanet, C. Ecoffet, and D. J. Lougnot, J. Polym. Sci., Part A: Polym. Chem. 37, 2075 (1999).

${ }^{30}$ G. Berkovic, V. Krongauz, and V. Weiss, Chem. Rev. (Washington, D.C.) 100, 1741 (2000).

${ }^{31}$ E. Mele, F. Di Benedetto, L. Persano, R. Cingolani, and D. Pisignano, Nano Lett. 5, 1915 (2005).

${ }^{32}$ D. Pisignano, L. Persano, R. Cingolani, G. Gigli, F. Babudri, G. M. Farinola, and F. Naso, Appl. Phys. Lett. 84, 1365 (2004).

${ }^{33}$ G. McHale, S. M. Rowan, and M. I. Newton, J. Phys. D 27, 2619 (1994).

${ }^{34}$ G. McHale and M. I. Newton, Colloids Surf., A 206, 193 (2002).

${ }^{35}$ G. McHale, N. J. Shirtcliffe, S. Aqil, C. C. Perry, and M. I. Newton, Phys. Rev. Lett. 93, 036102 (2004).

${ }^{36}$ Y. Abe, R. Nakao, T. Horii, S. Okada, and M. Irie, J. Photochem. Photobioí., A 95, 209 (1995). 